\title{
Development of electronics and data acquisition system for the J-PARC T59 (WAGASCI) experiment
}

\author{
Naruhiro CHIKUMA ${ }^{a *}$, F. Hosomi ${ }^{a}$, T.Koga $^{a}$, R.Tamura $^{a}$, M.Yokoyama $^{a}$, \\ M.Khabibullin $^{b}$, A.Khotjantsev ${ }^{b}$, A.Kostin $^{b}$, Y.Kudenko $^{b}$, A.Mefodiev $^{b}$, O.Mineev $^{b}$, \\ S.Suvorov $^{b}$, N.Yershov ${ }^{b}$, T.Kobayashi $^{c}$, S.Cao $^{c}$, T.Hayashino $^{d}$, A.Hiramoto $^{d}$, \\ A.K.Ichikawa ${ }^{d}$, T.Nakaya $^{d}$, K. Yasutome ${ }^{d}$, A.Bonnemaison ${ }^{e}$, R.Cornat $^{e}$, O.Drapier ${ }^{e}$, \\ O.Ferreira ${ }^{e}$, F.Gastaldi ${ }^{e}$, M.Gonin $^{e}$, J.Imber $^{e}$, M.Licciardi $^{e}$, F.Magniette $^{e}$, T.Mueller $^{e}$,

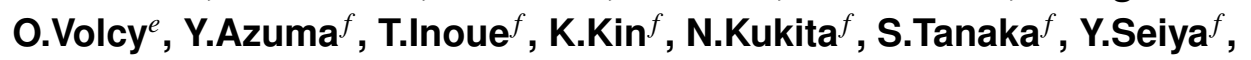 \\ K.Yamamoto ${ }^{f}$, A.Blondel ${ }^{g}$, F.Cadoux ${ }^{g}$, Y.Karadzhov ${ }^{g}$, Y.Favre ${ }^{g}$, E.Noah ${ }^{g}$ L.Nicola $^{g}$, \\ S.Parsa ${ }^{g}$ M.Rayner $^{g}$, Y.Hayato $^{h}$, A.Minamino ${ }^{i}$ \\ ${ }^{a}$ The University of Tokyo, ${ }^{b}$ Institute for Nuclear Research of the Russian Academy of Science \\ (INR), ${ }^{c}$ KEK, ${ }^{d}$ Kyoto University, ${ }^{e}$ Laboratoire Leprince-Ringuet (LLR), Ecole Polytechnique, \\ ${ }^{f}$ Osaka City University, ${ }^{g}$ University of Geneva, \\ ${ }^{h}$ Institute of Cosmic-Ray Research, University of Tokyo, ${ }^{i}$ Yokohama National University \\ E-mail: nchikuma@hep.phys.s.u-tokyo.ac.jp
}

\begin{abstract}
WAGASCI is a new experiment at J-PARC neutrino beamline, to measure the cross section ratio of charged current neutrino interaction on nucleus between water target and plastic target with the accuracy of a few percent. The detector adopts three-dimensional grid structure of 3-mmthick scintillator bars around water and plastic targets, to obtain large angular acceptance. As a photodetector a 32-channel arrayed MPPC has been developed for the WAGASCI detector. The WAGASCI detector has 1280 MPPC channels in total. Our front-end electronics is SPIROC2D, which is an auto-triggered, bi-gain, 36-channel ASIC, allowing to measure the charge from one to 2000 photoelectron and the time with 100ps step. It contains a 16-deep analog memory array, which allows to store 16 hits in an acquisition gate. The back-end boards control the data output from the front-end ASIC and reception of trigger signals for neutrino beam. The module construction has been completed and the data acquisition system has almost been completed, to be ready for the neutrino beam measurement from October 2017.
\end{abstract}

The European Physical Society Conference on High Energy Physics

5-12 July

Venice, Italy

* Speaker 


\section{Physics Motivation}

In the neutrino oscillation measurement in the T2K experiment, it has been required to better understand neutrino-nucleus interactions to suppress the systematic errors. Even if the systematic errors on the far detector, Super-Kamiokande, are suppressed by using constraints from measurements on the near detector, ND280, there are residual systematic errors on the neutrino beam flux and neutrino cross section, such as $4.8 \%(2.9 \%)$ on $v_{e}\left(v_{\mu}\right)$ events [1]. To constrain these errors, it is important to measure neutrino cross sections on water target and hydrocarbon target with large acceptance, for providing new information to improve the neutrino interaction model.

\section{WAGASCI experiment}

The WAGASCI experiment aims to measure the cross section ratio of charged current neutrino interaction on nucleus between water and hydrocarbon with $3 \%$ accuracy, and to measure differential cross section with large phase space acceptance, at J-PARC neutrino beamline. The detector contains both water target region and hydrocarbon target region, surrounded by muon range detectors as shown in Fig. 1. The muon range detectors contain side MRDs located at either side of the WAGASCI modules, and the Baby MIND downstream of these modules. Charged particles are detected by using 3-mm-thick plastic scintillator bars, which is aligned in a three-dimensional grid structure as shown in Fig. 2 By filling each cell with water or hydrocarbon target, this structure benefits from having about $80 \%$ target region in the fiducial volume, and from almost $4 \pi$ acceptance around the target. The location of the WAGASCI detector is on $1.5^{\circ}$ off-axis from the neutrino beam center, to achieve measurements with similar neutrino energy to that for $\mathrm{T} 2 \mathrm{~K}$ at $2.5^{\circ}$ off-axis.

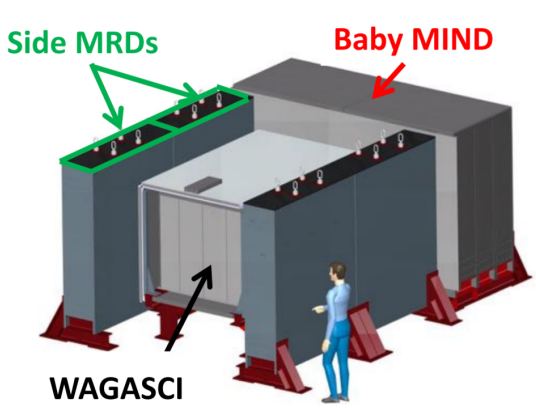

Figure 1: The configuration of the WAGASCI detectors.

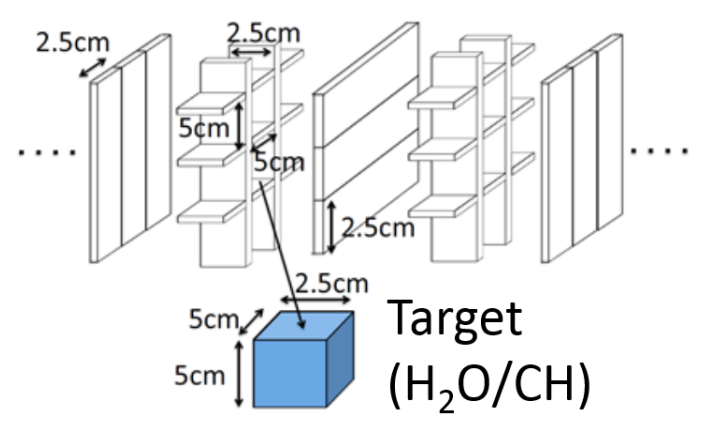

Figure 2: The three-dimensional grid-like structure of the plastic scintillator inside of the central detector.

\section{Electronics and data acquisition system}

The J-PARC neutrino beam has 2.48-second-period spill structure, and each spill contains 8 bunches with 581ns gaps. To readout 1280 channels of photodetector (MPPC) to the neutrino beam and to control them, a new electronics with a Silicon PM Integrated Read-Out Chip (SPIROC) has been developed. SPIROC is a 36-channel auto-triggered front-end ASIC, and is produced 
by OMEGA/IN2P3[2]. The adopted version in WAGASCI is SPIROC2D, which is capable of adjustment of MPPC gains, charge measurement with a wide dynamic range from one to 2,000 photoelectrons, and time measurement with about 100 ps step.

The whole electronics system of WAGASCI is shown in Fig. 3. The front-end board for WAGASCI, named ASU, has been developed with the SPIROC2D, and designed to directly attached to a 32-channel arrayed MPPC on the surface of the WAGASCI detector, as shown in Fig. 4 The ASU boards are connected in a daisy chain, and the data readout and the chip configuration are serially performed. The configuration of the SPIROC2D and the wrapping of the data are performed by the detector interface board (DIF), and one of back-end boards, GDCC, performs the data transfer between a data acquisition server and front-end boards. The whole system is synchronized to $50 \mathrm{MHz}$ clock generated on the other back-end board, CCC.

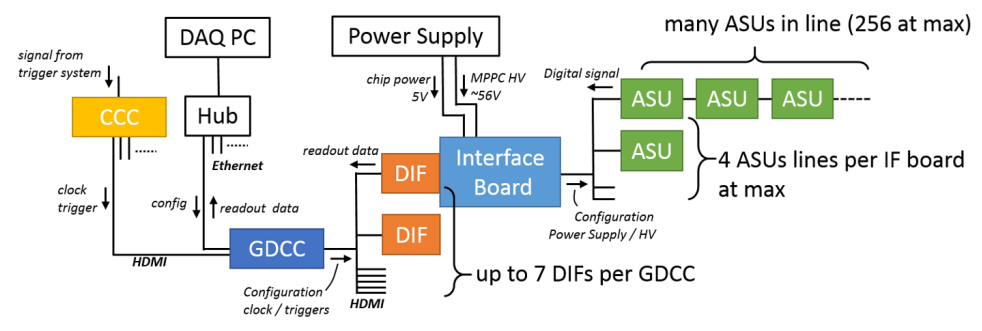

Figure 3: Front-end and back-end electronics boards.
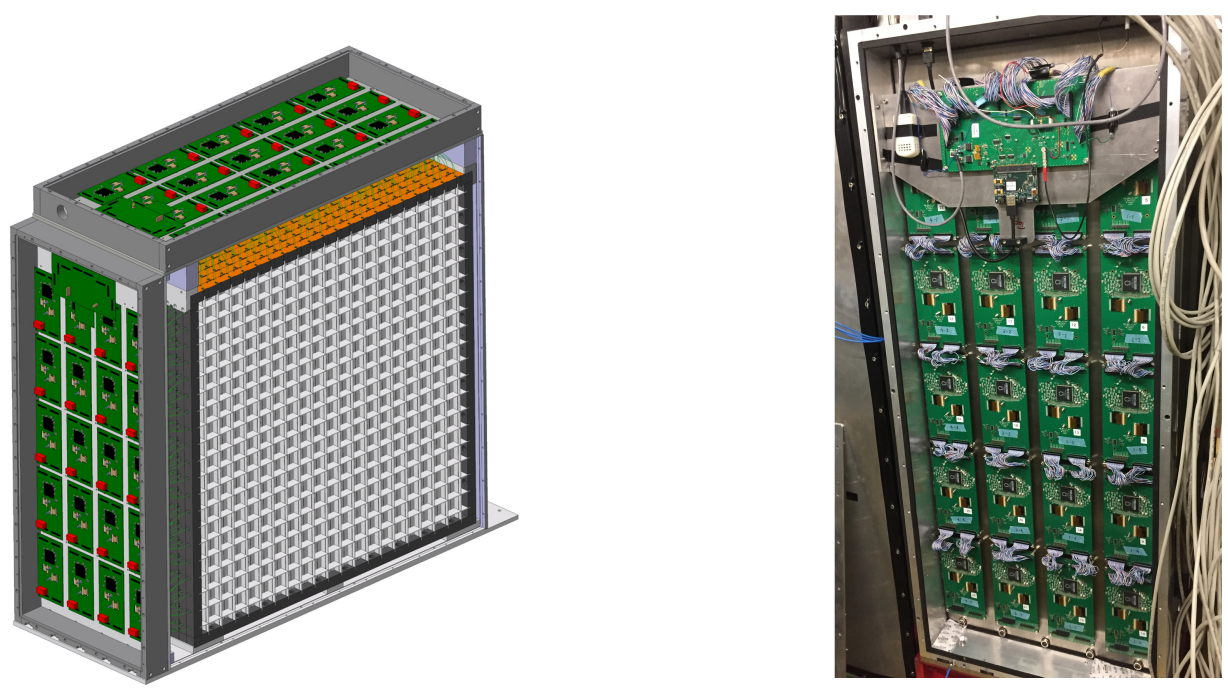

Figure 4: ASUs attached to the WAGASCI module.

\subsection{Triggering system}

The CCC board also receives trigger signals from the neutrino beamline. Firmware for a Spartan 6 FPGA has been developed to open an acquisition gate by receiving two different timing signals. The first beam timing signal (pre-beam trigger) arrives exactly $100 \mathrm{~ms}$ before the second 
one (beam trigger), and then about $30 \mu \mathrm{s}$ later then the beam trigger, neutrino beam arrives at the near detectors. The spill number information for tagging the event is also delivered from the beamline as 16-bit ECL signal, and received by a Xilinx Zynq-7000 development board. Firmware for the Zynq-7000 has been developed to receive the 16-bit ECL signal and to send it to GDCC board on Ethernet frame.

\section{Detector construction}

The WAGASCI detector has been constructed since the end of 2016, and completed at June 2016. The detector has already been installed into the T2K near detector hall in J-PARC, at August 2017, as shown in Fig. 5, Commissioning of the detector has been performed, and it is almost ready to start neutrino beam measurement from the middle of October 2017.

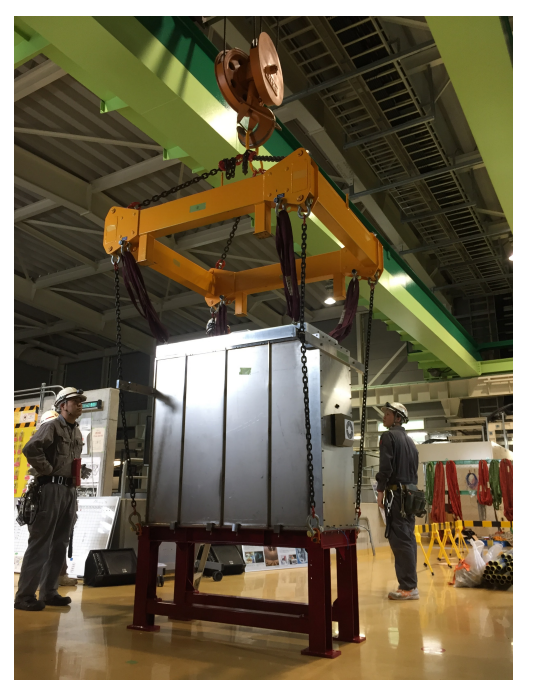

Figure 5: Installation of the WAGASCI module.

\section{Summary}

For the WAGASCI experiment, new electronics with SPIROC2D have been developed. The data acquisition system for the WAGASCI electronics has also been developed, and all the system are ready for the neutrino beam measurement. The commissioning of the detector and the data acquisition system has been performed, to start the neutrino beam data taking from October in 2017.

\section{References}

[1] K. Abe et al. (T2K Collaboration), arXiv:1707.01048 [hep-ex]

[2] http://omega.in2p3.fr/ 